\title{
Spin-orbit-coupled transport and spin torque in a ferromagnetic heterostructure
}

\author{
Xuhui Wang, ${ }^{*}$ Christian Ortiz Pauyac, and Aurélien Manchon ${ }^{\dagger}$ \\ King Abdullah University of Science and Technology (KAUST), Physical Science and Engineering Division, \\ Thuwal 23955-6900, Saudi Arabia
}

(Received 20 November 2013; revised manuscript received 27 January 2014; published 7 February 2014)

\begin{abstract}
Ferromagnetic heterostructures provide an ideal platform to explore the nature of spin-orbit torques arising from the interplay mediated by itinerant electrons between a Rashba-type spin-orbit coupling and a ferromagnetic exchange interaction. For such a prototypic system, we develop a set of coupled diffusion equations to describe the diffusive spin dynamics and spin-orbit torques. We characterize the spin torque and its two prominentout-of-plane and in-plane - components for a wide range of relative strength between the Rashba coupling and ferromagnetic exchange. The symmetry and angular dependence of the spin torque emerging from our simple Rashba model is in an agreement with experiments. The spin diffusion equation can be generalized to incorporate dynamic effects such as spin pumping and magnetic damping.
\end{abstract}

DOI: 10.1103/PhysRevB.89.054405

PACS number(s): 75.60.Jk, 72.25.Ba, 72.25.Rb, 75.70.Tj

\section{INTRODUCTION}

Spin-orbit coupling is a key mechanism in many prominent physical phenomena ranging from the electrically generated bulk spin polarization $[1,2]$ to the dissipationless spin current in bulk semiconductors [3] to the spin-Hall effect in metals [4] and two-dimensional electron gas [5,6]. In searching for an efficient mechanism for magnetization switching, interplay between spin-orbit coupling and magnetism $[7,8]$ has brought a new member to the spin-transfer torque community $[9,10]$, i.e., the spin-orbit torque. Diluted magnetic semiconductors provide an ideal platform to theoretically [11] and experimentally [12-14] study the current-driven magnetization dynamics induced by spin-orbit torques. Simply speaking, spin-orbit torque operates through the competition between the exchange and spin-orbit fields in polarizing the itinerant electrons (or holes) and gives rise to a torque on the ferromagnetic order parameter. When it comes to magnetization switching, the advantage of spin-orbit torque over the conventional one is clear: there is no need to employ a separate ferromagnet as polarizer.

Recent experiments on current-driven magnetization dynamics performed in multilayer systems [15-19] have also achieved current-induced switching in a single ferromagnet film sandwiched between a heavy metal and metal oxide, which indicates the presence of spin-orbit coupling and therefore spin-orbit torque as a potential driving force. These systems-mostly consisting of conducting interfaces between ferromagnetic metal films and heavy metals (or metal oxides) - are nowadays often referred to as ferromagnetic heterostructures. A viable candidate believed to exist in such structures is the Rashba-type spin-orbit interaction due to inversion symmetry breaking [20]. Theoretical efforts are made to uncover dominant components of the spin-orbit torque induced by the Rashba coupling [21-25]. They usually treat the coexistence of ferromagnetism and spin-orbit coupling as an intrinsic property. The underlying physics is fairly simple and intuitive: when a charge current is applied in the

\footnotetext{
*xuhui.wang@kaust.edu.sa

†aurelien.manchon@kaust.edu.sa
}

structure, the Rashba spin-orbit coupling creates an effective magnetic field (coined as the Rashba field $\boldsymbol{B}_{R}$ ); so long as $\boldsymbol{B}_{R}$ polarizes charge carriers to the direction that is misaligned with the magnetization direction $\boldsymbol{m}$, a spin torque emerges to act on the magnetization and to induce switching. This torque, named as Rashba spin-orbit torque or Rashba torque, has gained much attention from academia as well as industries and is exactly the central topic of this paper. Both theories and experiments have shown that the Rashba torque shall, in general, comprise two major components, i.e., a fieldlike torque (or in-plane component) and a dampinglike torque (or out-of-plane component). More recently, the symmetry of spin-orbit torque has been scrutinized experimentally. The experiments by Garello et al. reveal an intriguing yet complex angular dependence on the magnetization direction [26]. This observation challenges the commonly accepted form described by in-plane and out-of-plane components.

In this paper, we provide a systematic theoretical study on the spin-orbit torque and spin dynamics in a ferromagnetic ultrathin film without structure inversion symmetry. We construct a simple two-dimensional model that accommodates both a Rashba spin-orbit coupling and an exchange interaction. For the Rashba torque, we propose a general form that not only contains the in-plane and out-of-plane components but also possesses symmetry and complex angular dependence supported by experiments. In Sec. II, we employ the quantum kinetic equation to derive coupled diffusion equations for the charge and spin densities. We account for the fact that Rashba coupling not only produces an effective magnetic field but also induces spin relaxation through the D'yakonov-Perel mechanism [27] that is dominant in a quasi-two-dimensional system. In the absence of magnetism, analytical and numerical solutions in Sec. III A are able to describe the spin-galvanic and spin-Hall effects. We demonstrate in Sec. III B that the diffusion equation provides a coherent framework to describe the spin dynamics in a ferromagnetic metal. Section IV employs the spin diffusion equation given in Sec. II to analyze the general symmetry properties and angular dependence of the Rashba torque in the limits of both a weak and strong spin-orbit coupling. We are able to provide for the spin-orbit torque an angular dependence that agrees well with recent experiments. In Sec. V, we evaluate the spin density and 
Rashba torque numerically for a wide range of relative strength between the Rashba coupling and exchange splitting. In Sec. VI, we further show the formulation proposed in Sec. II can be generalized to describe spin pumping and magnetic damping by an inclusion of temporal and spatial variations of ferromagnetic order parameter. We find agreement with earlier results approached using other methods. Section VII discusses the validity of the Rashba model and outlines a brief comparative study between the Rashba torque and spin-Hall effect-induced torque. Section VIII concludes the article.

Nevertheless, we emphasize that we make no attempt to argue that the Rashba model provides the ultimate answer to the spin-orbit torque in ferromagnetic heterostructures. Spin-Hall effect [4] in the nonmagnetic metal layer provides an alternative explanation to several experiments [28]. However, we must admit, as Haney et al. have pointed out, both explanations have their strength and weakness [29]. Despite the limitations, the results presented here and their agreement with experiments lead us to believe such a simple model does shed light on the nature of the spin-orbit torque in ferromagnetic thin films.

\section{FROM HAMILTONIAN TO DIFFUSION EQUATION}

In Fig. 1, we sketch a schematic view of a cross section of a typical ferromagnetic heterostructure under investigation: a ferromagnetic ultrathin metal film (rolled out in the $x-y$ plane) is sandwiched by a heavy metal layer and an oxide; two asymmetric interfaces provide a weak confinement in the $z$ direction, along which the inversion symmetry is broken. The potential gradient across the interface generates a Rashba spinorbit coupling [20]. Without loss of generality, our starting point is therefore a simplified quasi-two-dimensional singleparticle Hamiltonian ( $\hbar=1$ is assumed throughout),

$$
\hat{H}=\frac{\hat{\boldsymbol{k}}^{2}}{2 m}+\alpha \hat{\boldsymbol{\sigma}} \cdot(\hat{\boldsymbol{k}} \times \hat{z})+\frac{1}{2} \Delta_{x c} \hat{\boldsymbol{\sigma}} \cdot \boldsymbol{m}+\hat{H}^{i},
$$

for an electron with momentum $\hat{\boldsymbol{k}}$. In Eq. (1), $\hat{\boldsymbol{\sigma}}$ is the Pauli matrix, $m$ the effective mass, and $\boldsymbol{m}$ the magnetization direction. The ferromagnetic exchange splitting is given by $\Delta_{x c}$ and $\alpha$ represents the Rashba constant (parameter). The Hamiltonian $\hat{H}^{i}=\sum_{j=1}^{N} V\left(\boldsymbol{r}-\boldsymbol{r}_{j}\right)$ accounts for all nonmagnetic impurity scattering potentials $V(\boldsymbol{r})$ localized at $\boldsymbol{r}_{j}$. Throughout the

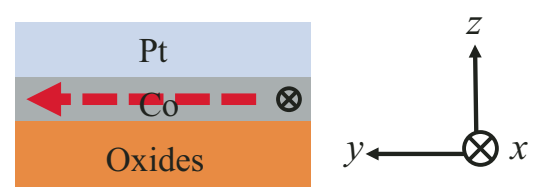

FIG. 1. (Color online) Schematic view of the cross section of a typical ferromagnetic heterostructure that accommodates both a Rashba spin-orbit coupling and an exchange interaction. An ultrathin ferromagnetic metal film (e.g., Co) is sandwiched between an oxide (e.g., $\mathrm{AlO}_{x}$ ) and a heavy metal layer (e.g., Pt or Ta). A charge current is injected into the ferromagnetic layer along the $\hat{\boldsymbol{x}}$ direction. The dashed red arrow points to the direction of the effective Rashba field. We shall note that the system is not isolated but connected to an external source and drain. following discussion, we assume that the exchange interaction and spin-orbit splitting are smaller than the Fermi energy, while leaving the ratio of the spin-orbit coupling to the exchange interaction arbitrary.

Before we proceed to detailed discussion, we clarify the validity of such a quasi-two-dimensional model. In principle, carrier transport in the system under consideration is a threedimensional phenomenon in which size effect may arise. Here, our quasi-two-dimensional Rashba model assumes a direct coupling between the exchange and effective Rashba field and is thus requiring ultrathin layers in which diffusive motion normal to the thin film plane can be neglected. Haney et al. have recently conducted a thorough discussion using a three-dimensional semiclassical Boltzmann description in a bilayer structure augmented by an interfacial Rashba spin-orbit coupling [29]. Their results are consistent with the ones obtained from quasi-two-dimensional transport modeled in Refs. [21-25,30].

To derive a diffusion equation for the nonequilibrium charge and spin densities, we apply the Keldysh formalism [31]. We use the Dyson equation, in a two-by-two spin space, to obtain a kinetic equation that assembles the retarded (advanced) Green's function $\hat{G}^{R}\left(\hat{G}^{A}\right)$, the Keldysh component of the Green function $\hat{G}^{K}$, and the self-energy $\hat{\Sigma}^{K}$, i.e.,

$$
\left[\hat{G}^{R}\right]^{-1} \hat{G}^{K}-\hat{G}^{K}\left[\hat{G}^{A}\right]^{-1}=\hat{\Sigma}^{K} \hat{G}^{A}-\hat{G}^{R} \hat{\Sigma}^{K},
$$

where all Green's functions are full functions with interactions taken care of by the self-energies $\hat{\Sigma}^{R, A, K}$. The retarded (advanced) Green's function in momentum and energy space is

$$
\hat{G}^{R(A)}(\boldsymbol{k}, \epsilon)=\frac{1}{\epsilon-\epsilon_{\boldsymbol{k}}-\hat{\boldsymbol{\sigma}} \cdot \boldsymbol{b}(\boldsymbol{k})-\hat{\Sigma}^{R(A)}(\boldsymbol{k}, \epsilon)},
$$

where $\epsilon_{\boldsymbol{k}}=\boldsymbol{k}^{2} /(2 m)$ is the single-particle energy. The impurity scattering has been taken into account by the self-energy, as to be shown below. We have introduced a $\boldsymbol{k}$-dependent total effective field $\boldsymbol{b}(\boldsymbol{k})=\Delta_{x c} \boldsymbol{m} / 2+\alpha(\boldsymbol{k} \times \hat{\boldsymbol{z}})$ with magnitude $b_{k}=\left|\Delta_{x c} \boldsymbol{m} / 2+\alpha(\boldsymbol{k} \times \hat{z})\right|$ and direction $\hat{\boldsymbol{b}}=\boldsymbol{b}(\boldsymbol{k}) / b_{k}$.

We neglect localization effects and electron-electron interactions and assume a short-range $\delta$-function type impurity scattering potential. At a low impurity concentration and a weak coupling to electrons, a second-order Born approximation is justified [31], i.e., the self-energy due to impurity scattering is [32]

$$
\hat{\Sigma}^{K, R, A}\left(\boldsymbol{r}, \boldsymbol{r}^{\prime}\right)=\frac{\delta\left(\boldsymbol{r}, \boldsymbol{r}^{\prime}\right)}{m \tau} \hat{G}^{K, R, A}(\boldsymbol{r}, \boldsymbol{r}),
$$

where the momentum relaxation time is given by

$$
\frac{1}{\tau} \approx 2 \pi \int \frac{d^{2} \boldsymbol{k}^{\prime}}{(2 \pi)^{2}}\left|V\left(\boldsymbol{k}-\boldsymbol{k}^{\prime}\right)\right|^{2} \delta\left(\epsilon_{\boldsymbol{k}}-\epsilon_{\boldsymbol{k}^{\prime}}\right) .
$$

$V(\boldsymbol{k})$ is the Fourier transform of the scattering potential and the magnitude of $\boldsymbol{k}$ and $\boldsymbol{k}^{\prime}$ is evaluated at Fermi vector $k_{F}$.

The quasiclassical distribution function $\hat{g} \equiv \hat{g}_{\boldsymbol{k}, \epsilon}(T, \boldsymbol{R})$, defined as the Wigner transform of the Keldysh function $\hat{G}^{K}\left(\boldsymbol{r}, t ; \boldsymbol{r}^{\prime}, t^{\prime}\right)$, is obtained by integrating out the relative spatial-temporal coordinates while retaining the center-ofmass ones $\boldsymbol{R}=\left(\boldsymbol{r}+\boldsymbol{r}^{\prime}\right) / 2$ and $T=\left(t+t^{\prime}\right) / 2$. The spatial profile of the quasiclassical distribution function is considered smooth on the scale of Fermi wavelength; we may thus apply 
the gradient expansion technique on Eq. (2) [33], which gives us a transport equation for macroscopic quantities. Under the gradient expansion, the left-hand side of Eq. (2) becomes

$$
\begin{aligned}
{\left[\hat{G}^{R}\right]^{-1} \hat{G}^{K}-\hat{G}^{K}\left[\hat{G}^{A}\right]^{-1} \approx } & {[\hat{g}, \hat{\boldsymbol{\sigma}} \cdot \boldsymbol{b}(\boldsymbol{k})]+\frac{i}{\tau} \hat{g}+i \frac{\partial \hat{g}}{\partial T} } \\
& +\frac{i}{2}\left\{\frac{\boldsymbol{k}}{m}+\alpha(\hat{z} \times \hat{\boldsymbol{\sigma}}), \nabla_{\boldsymbol{R}} \hat{g}\right\},
\end{aligned}
$$

where $\{\cdot, \cdot\}$ is the anticommutator. The relaxation time approximation renders the right-hand side of Eq. (2) as

$$
\begin{aligned}
& \hat{\Sigma}^{K} \hat{G}^{A}-\hat{G}^{R} \hat{\Sigma}^{K} \\
& \quad \approx \frac{1}{\tau}\left[\hat{\rho}(\epsilon, T, \boldsymbol{R}) \hat{G}^{A}(\boldsymbol{k}, \epsilon)-\hat{G}^{R}(\boldsymbol{k}, \epsilon) \hat{\rho}(\epsilon, T, \boldsymbol{R})\right],
\end{aligned}
$$

where we have introduced the density matrix by integrating out $\boldsymbol{k}^{\prime}$ in $\hat{g}$, i.e.,

$$
\hat{\rho}(\epsilon, T, \boldsymbol{R})=\frac{1}{2 \pi \mathcal{N}} \int \frac{d^{2} \boldsymbol{k}^{\prime}}{(2 \pi)^{2}} \hat{g}_{\boldsymbol{k}^{\prime}, \epsilon}(T, \boldsymbol{R}),
$$

where $\mathcal{N}$ is the density of states for one spin specie.

For the convenience of discussion, the time variable is changed from $T$ to $t$. At this stage, we have a kinetic equation depending on $\hat{\rho}$ and $\hat{g}$

$$
\begin{aligned}
& i[\hat{\boldsymbol{\sigma}} \cdot \boldsymbol{b}(\boldsymbol{k}), \hat{g}]+\frac{1}{\tau} \hat{g}+\frac{\partial \hat{g}}{\partial t}+\frac{1}{2}\left\{\frac{\boldsymbol{k}}{m}+\alpha(\hat{z} \times \hat{\boldsymbol{\sigma}}), \nabla_{\boldsymbol{R}} \hat{g}\right\} \\
& \quad=\frac{i}{\tau}\left[\hat{G}^{R}(\boldsymbol{k}, \epsilon) \hat{\rho}(\epsilon)-\hat{\rho}(\epsilon) \hat{G}^{A}(\boldsymbol{k}, \epsilon)\right] .
\end{aligned}
$$

We perform a Fourier transformation on temporal variable to the frequency domain $\omega$, which leads to

$$
\Omega \hat{g}-b_{k}\left[\hat{U}_{k}, \hat{g}\right]=i \hat{K},
$$

where $\Omega=\omega+i / \tau$ and the operator $\hat{U}_{k} \equiv \hat{\boldsymbol{\sigma}} \cdot \hat{\boldsymbol{b}}$ satisfies $\hat{U}_{k} \hat{U}_{k}=1$. The right-hand side of Eq. (10) is partitioned according to

$$
\begin{aligned}
\hat{K}= & \underbrace{\frac{i}{\tau}\left[\hat{G}^{R}(\boldsymbol{k}, \epsilon) \hat{\rho}(\epsilon)-\hat{\rho}(\epsilon) \hat{G}^{A}(\boldsymbol{k}, \epsilon)\right]}_{\hat{K}^{(0)}} \\
& +\underbrace{-\frac{1}{2}\left\{\frac{\boldsymbol{k}}{m}+\alpha(\hat{z} \times \hat{\boldsymbol{\sigma}}), \nabla_{\boldsymbol{R}} \hat{g}\right\}}_{\hat{K}^{(1)}},
\end{aligned}
$$

where $\hat{K}^{(0)}$ contributes to the lowest-order solution to $\hat{g}$ and the gradient correction $\hat{K}^{(1)}$ is treated as a perturbation. Both functions $\hat{g}$ and $\hat{\rho}$ are in the frequency domain.

Equation (10) is solved formally to give a solution to $\hat{g}$ :

$\hat{g}=i \frac{\left(2 b_{k}^{2}-\Omega^{2}\right) \hat{K}+2 b_{k}^{2} \hat{U}_{k} \hat{K} \hat{U}_{k}-\Omega b_{k}\left[\hat{U}_{k}, \hat{K}\right]}{\Omega\left(4 b_{k}^{2}-\Omega^{2}\right)} \equiv \mathscr{L}[\hat{K}]$.

An iteration procedure to solve Eq. (12) has been outlined in Ref. [32]. We adopt the procedures here: according to the partition scheme of $\hat{K}$, we use $\hat{K}^{(0)}$ to obtain the zeroth order approximation given by $\hat{g}^{(0)} \equiv \mathscr{L}\left[\hat{K}^{(0)}(\hat{\rho})\right]$, which replaces $\hat{g}$ in $\hat{K}^{(1)}$ to generate a correction due to the gradient term, i.e., $\hat{K}^{(1)}\left(\hat{g}^{(0)}\right)$; we further insert $\hat{K}^{(1)}\left(\hat{g}^{(0)}\right)$ back to Eq. (12) to obtain a correction $\mathscr{L}\left[\hat{K}^{(1)}\left(\hat{g}^{(0)}\right)\right]$; then we have the first order approximation to the quasiclassical distribution function,

$$
\hat{g}^{(1)}=\hat{g}^{(0)}+\mathscr{L}\left[\hat{K}^{(1)}\left(\hat{g}^{(0)}\right)\right] .
$$

The above procedure is repeated to a desired order using

$$
\hat{g}^{(n)}=\hat{g}^{(n-1)}+\mathscr{L}\left[\hat{K}^{(1)}\left(\hat{g}^{(n-1)}\right)\right] .
$$

In this paper, the second order approximation is sufficient. The full expression of the second order approximation for $\hat{g}$ is tedious thus to be excluded in the following. The diffusion equation is derived by an angle averaging in momentum space, which allows all terms that are of odd order in $k_{i}(i=x, y)$ to vanish while the combinations such as $k_{i} k_{j}$ contribute to the averaging by a factor $k_{F}^{2} \delta_{i j}$ [33].

A Fourier transform from frequency domain back to the real time brings us a diffusionlike equation for the density matrix,

$$
\begin{aligned}
\frac{\partial}{\partial t} \hat{\rho}(t)= & D \nabla^{2} \hat{\rho}-\frac{1}{\tau_{\mathrm{dp}}} \hat{\rho}+\frac{1}{2 \tau_{\mathrm{dp}}}(\hat{z} \times \hat{\boldsymbol{\sigma}}) \cdot \hat{\rho}(\hat{z} \times \hat{\boldsymbol{\sigma}}) \\
& +i C[\hat{z} \times \hat{\boldsymbol{\sigma}}, \nabla \hat{\rho}]-B\{\hat{z} \times \hat{\boldsymbol{\sigma}}, \nabla \hat{\rho}\} \\
& +\Gamma\left[(\boldsymbol{m} \times \nabla)_{z} \hat{\rho}-\hat{\boldsymbol{\sigma}} \cdot \boldsymbol{m} \nabla \hat{\rho} \cdot(\hat{z} \times \hat{\boldsymbol{\sigma}})\right. \\
& -(\hat{z} \times \hat{\boldsymbol{\sigma}}) \cdot \nabla \hat{\rho} \hat{\boldsymbol{\sigma}} \cdot \boldsymbol{m}]+\frac{1}{2 \tau_{\varphi}}(\hat{\boldsymbol{\sigma}} \cdot \boldsymbol{m} \hat{\rho} \hat{\boldsymbol{\sigma}} \cdot \boldsymbol{m}-\hat{\rho}) \\
& -i \tilde{\Delta}_{x c}[\hat{\boldsymbol{\sigma}} \cdot \boldsymbol{m}, \hat{\rho}]-2 R\left\{\hat{\boldsymbol{\sigma}} \cdot \boldsymbol{m},(\boldsymbol{m} \times \nabla)_{z} \hat{\rho}\right\},
\end{aligned}
$$

where $\hat{\rho}$ assumes an energy dependence $\hat{\rho} \equiv \hat{\rho}(\epsilon)$. The subscript is omitted for the brevity of notation. In a twodimensional system, the diffusion constant $D=\tau v_{F}^{2} / 2$ is given in terms of Fermi velocity $v_{F}$ and momentum relaxation time $\tau$. The renormalized exchange splitting reads $\tilde{\Delta}_{x c}=$ $\left(\Delta_{x c} / 2\right) /\left(4 \varsigma^{2}+1\right)$, where $\varsigma^{2}=\left(\Delta_{x c}^{2} / 4+\alpha^{2} k_{F}^{2}\right) \tau^{2}$. The other parameters are given by

$$
\begin{aligned}
C & =\frac{\alpha k_{F} v_{F} \tau}{\left(4 \varsigma^{2}+1\right)^{2}}, \quad \Gamma=\frac{\alpha \Delta_{x c} v_{F} k_{F} \tau^{2}}{2\left(4 \varsigma^{2}+1\right)^{2}}, \\
R & =\frac{\alpha \Delta_{x c}^{2} \tau^{2}}{2\left(4 \varsigma^{2}+1\right)}, \quad B=\frac{2 \alpha^{3} k_{F}^{2} \tau^{2}}{4 \varsigma^{2}+1}, \\
\frac{1}{\tau_{\mathrm{dp}}} & =\frac{2 \alpha^{2} k_{F}^{2} \tau}{4 \varsigma^{2}+1}, \quad \frac{1}{\tau_{\varphi}}=\frac{\Delta_{x c}^{2} \tau}{4 \varsigma^{2}+1} .
\end{aligned}
$$

$\tau_{\mathrm{dp}}$ is the relaxation time due to the D'yakonov-Perel mechanism [1] and $\tau_{\Delta} \equiv 1 / \Delta_{x c}$ sets the time scale for the coherent precession of the spin density around the magnetization. Equation (15) is valid in the dirty limit $\varsigma \ll 1$, which enables the approximation $1+4 \varsigma^{2} \approx 1$. The charge density $n$ and spin density $S$ are introduced by a vector decomposition of the density matrix $\hat{\rho}_{\epsilon}=n_{\epsilon} / 2+S_{\epsilon} \cdot \hat{\sigma}$. In real experiments $[15,18,19]$, spin transport in a ferromagnetic film experiences random magnetic scatterers, for which we introduce phenomenologically an isotropic spin-flip relaxation $S / \tau_{s f}$.

After an integration over energy $\epsilon$, i.e., $n=\mathcal{N} \int d \epsilon n_{\epsilon}$ and $S=\mathcal{N} \int d \epsilon S_{\epsilon}$, we obtain a set of diffusion equations for the charge and spin densities, i.e.,

$$
\frac{\partial n}{\partial t}=D \nabla^{2} n+B \nabla_{z} \cdot S+\Gamma \nabla_{z} \cdot \boldsymbol{m} n+R \nabla_{z} \cdot \boldsymbol{m}(\boldsymbol{S} \cdot \boldsymbol{m}),
$$


where $\nabla_{z} \equiv \hat{z} \times \nabla$ and

$$
\begin{aligned}
\frac{\partial \boldsymbol{S}}{\partial t}= & D \nabla^{2} \boldsymbol{S}-\frac{\boldsymbol{S}}{\tau_{s f}}-\frac{\boldsymbol{S}+S_{z} z}{\tau_{\mathrm{dp}}}-\frac{1}{\tau_{\Delta}} \boldsymbol{S} \times \boldsymbol{m}-\frac{\boldsymbol{m} \times(\boldsymbol{S} \times \boldsymbol{m})}{\tau_{\varphi}} \\
& +B \nabla_{z} n+2 C \nabla_{z} \times \boldsymbol{S}+2 R\left(\boldsymbol{m} \cdot \nabla_{z} n\right) \boldsymbol{m} \\
& +\Gamma\left[\boldsymbol{m} \times\left(\nabla_{z} \times \boldsymbol{S}\right)+\nabla_{z} \times(\boldsymbol{m} \times \boldsymbol{S})\right] .
\end{aligned}
$$

The anisotropy in spin relaxation is embedded naturally in our model: the spin density components $S_{x} \hat{\boldsymbol{x}}$ and $S_{y} \hat{\boldsymbol{y}}$ are relaxed at a rate $1 / \tau_{\mathrm{dp}}+1 / \tau_{s f}$, while $S_{z} \hat{z}$ is submitted to a higher rate $2 / \tau_{\mathrm{dp}}+1 / \tau_{s f}$.

Equations (16) and (17) comprise one of the most important results in this paper. For a broad range of the relative strength between the spin-orbit coupling and exchange splitting $\alpha k_{F} / \Delta_{x c}$, Eqs. (16) and (17) not only describe the spin dynamics in a ferromagnetic film but also capture the symmetry of the spin-orbit torque. When the magnetism vanishes $\Delta_{x c}=0$, the $B$ term behaves as a source that generates spin density electrically [2,32]. On the other hand, when the Rashba spin-orbit coupling is absent $(\alpha=0)$, the first two lines in Eq. (17) describe a diffusive motion of spin density in a ferromagnetic metal, which, to be shown in the next section, agrees with early results [34]. The $C$ term describes the coherent precession of the spin density around the effective Rashba field. The spin density induced by the Rashba field precesses around the exchange field, which is described by the $\Gamma$ term, and is thus at a higher order than the $C$ term in the dirty limit, for $\Gamma=\Delta_{x c} \tau C / 2$. The $R$ term contributes to a magnetization renormalization.

We shall assign a proper physical meaning to the transverse spin dephasing time $\tau_{\varphi}$ defined in this paper. Here, the dephasing time $\tau_{\varphi}$ is different from the transverse spin scattering time in, for example, Eq. (34) in Ref. [35] that describes the disorder contribution to the transverse spin scattering. $\tau_{\varphi}$ rather contributes to the transverse spin conductivity $\sigma_{\operatorname{tr}} \propto \frac{n}{m} \tau_{\varphi}$ and it plays the same role to the transverse component of spin current as the momentum relaxation time $\tau$ does in the ordinary Drude conductivity. In fact, $\tau_{\varphi}$ agrees with the calculation in Ref. [35] when the weak ferromagnet limit is taken, i.e., $\mu_{\uparrow} \approx \mu_{\downarrow} \approx \epsilon_{F}$ and $v_{\uparrow} \approx v_{\downarrow} \approx \mathcal{N}$.

\section{SPIN TRANSPORT}

\section{A. Edelstein effect and spin-Hall effect: Vanishing magnetism}

An electrically generated nonequilibrium spin density due to spin-orbit coupling [2] can be extracted from Eq. (17) by setting the exchange interaction to zero $\Delta_{x c}=0$. If we keep D'yakonov-Perel as the only spin relaxation mechanism and let $\tau_{s f}=\infty$, Eq. (17) reads

$$
D \nabla^{2} S-\frac{S+S_{z} \hat{z}}{\tau_{\mathrm{dp}}}+2 C \nabla_{z} \times S+B \nabla_{z} n=0,
$$

which also describes the spin-Hall effect in the diffusive regime $[32,36,37]$. Besides the spin relaxation, the second term in Eq. (18), the spin dynamics is controlled by two competing effects: the spin precession around the Rashba field (third term) and the electrical spin generation first pointed out by Edelstein [2]. In an infinite medium where a charge current is flowing along the $\hat{\boldsymbol{x}}$ direction, Eq. (18) leads to a solution

$$
\boldsymbol{S}=e E \tau_{\mathrm{dp}} B \frac{n}{\epsilon_{F}} \hat{\boldsymbol{y}}=\frac{e E \zeta}{\pi v_{F}} \hat{\boldsymbol{y}}
$$

where only the linear term in electric field has been retained. On the right-hand side, we have used the charge density in a two-dimensional system $n=k_{F}^{2} /(2 \pi)$ and introduced the parameter $\zeta=\alpha k_{F} \tau$ as used in Ref. [32]. In the presence of a weak spin-orbit coupling, only the spin precession term survives; the electrical spin generation dominates when the coupling is strong.

\section{B. Spin diffusion in a ferromagnet}

Spin diffusion in a ferromagnet has been discussed actively in the field of spintronics [34,35,38,39]. In this section we show explicitly that, by suppressing the Rashba spin-orbit coupling, Eq. (17) is able to describe spin diffusion in a ferromagnetic metal. A vanishing Rashba spin-orbit coupling means $\alpha=0$ and Eq. (17) reduces to

$$
\frac{\partial \boldsymbol{S}}{\partial t}=D \nabla^{2} \boldsymbol{S}+\frac{\boldsymbol{m} \times \boldsymbol{S}}{\tau_{\Delta}}-\frac{\boldsymbol{S}}{\tau_{s f}}-\frac{\boldsymbol{m} \times(\boldsymbol{S} \times \boldsymbol{m})}{\tau_{\varphi}},
$$

This equation only differs from the result of Ref. [38] by a dephasing term of the transverse component of the spin density.

In a ferromagnetic metal, we may divide the spin density into a longitudinal component that follows the magnetization direction adiabatically, and a deviation that is perpendicular to the magnetization, i.e., $\boldsymbol{S}=s_{0} \boldsymbol{m}+\delta \boldsymbol{S}$, where $s_{0}$ is the local equilibrium spin density. Such a partition, after restoring the electric field by $\boldsymbol{\nabla} \rightarrow \boldsymbol{\nabla}+\left(e / \epsilon_{F}\right) \boldsymbol{E}$, gives rise to

$$
\begin{aligned}
\frac{\partial}{\partial t} \delta \boldsymbol{S}+\frac{\partial}{\partial t} s_{0} \boldsymbol{m}= & s_{0} D \nabla^{2} \boldsymbol{m}+D \nabla^{2} \delta \boldsymbol{S}+D e P_{F} \mathcal{N}_{F} \boldsymbol{E} \cdot \nabla \boldsymbol{m} \\
& -\frac{\delta \boldsymbol{S}}{\tau_{s f}}-\frac{s_{0} \boldsymbol{m}}{\tau_{s f}}-\frac{\delta \boldsymbol{S}}{\tau_{\varphi}}+\frac{1}{\tau_{\Delta}} \boldsymbol{m} \times \delta \boldsymbol{S}, \quad \text { (21) }
\end{aligned}
$$

where the magnetic order parameter is allowed to be spatial dependent $\boldsymbol{m}=\boldsymbol{m}(\boldsymbol{r}, t)$. We introduce $P_{F}$ the spin polarization and $\mathcal{N}_{F}$ the density of state; both are at Fermi energy $\epsilon_{F}$.

In a smooth magnetic texture, the characteristic length scale of the magnetic profile is much larger than the length scale of electron transport; we discard the contribution $D \nabla^{2} \delta \boldsymbol{S}$ [34]. The diffusion of the equilibrium spin density follows $s_{0} D \nabla^{2} \boldsymbol{m} \approx s_{0} \boldsymbol{m} / \tau_{s f}$. In this paper, we retain only terms that are at first order in temporal derivative, which simplifies Eq. (21) to

$$
\xi \frac{\delta \boldsymbol{S}}{\tau_{\Delta}}-\frac{\boldsymbol{m} \times \delta \boldsymbol{S}}{\tau_{\Delta}}=D e P_{F} \mathcal{N}_{F} \boldsymbol{E} \cdot \nabla \boldsymbol{m}-s_{0} \frac{\partial \boldsymbol{m}}{\partial t} .
$$

The last equation can be solved exactly to show

$$
\begin{aligned}
\delta \boldsymbol{S}= & \frac{\tau_{\Delta}}{1+\xi^{2}}\left[\frac{P_{F}}{e} \boldsymbol{m} \times\left(\boldsymbol{j}_{e} \cdot \nabla\right) \boldsymbol{m}+\xi \frac{P_{F}}{e}\left(\boldsymbol{j}_{e} \cdot \nabla\right) \boldsymbol{m}\right. \\
& \left.-s_{0} \boldsymbol{m} \times \frac{\partial \boldsymbol{m}}{\partial t}-\xi s_{0} \frac{\partial \boldsymbol{m}}{\partial t}\right],
\end{aligned}
$$


where $\xi=\tau_{\Delta}\left(1 / \tau_{s f}+1 / \tau_{\varphi}\right)$ and the electric current $\boldsymbol{j}_{e}=$ $e^{2} n \tau \boldsymbol{E} / m$ is given in terms of electron density $n$. Apart from the transverse dephasing time absorbed in parameter $\xi$, the nonequilibrium spin density Eq. (23) agrees with Eq. (8) in Ref. [34]. Given the knowledge of the nonequilibrium spin density, the spin torque, defined as

$$
\boldsymbol{T}=-\frac{1}{\tau_{\Delta}} \boldsymbol{m} \times \delta \boldsymbol{S}+\frac{1}{\tau_{\varphi}} \delta \boldsymbol{S},
$$

is given by

$$
\begin{aligned}
\boldsymbol{T}= & -(1-\xi \tilde{\beta}) s_{0} \frac{\partial \boldsymbol{m}}{\partial t}+\tilde{\beta} s_{0} \boldsymbol{m} \times \frac{\partial \boldsymbol{m}}{\partial t} \\
& +(1-\xi \tilde{\beta}) \frac{P_{F}}{e}\left(\boldsymbol{j}_{e} \cdot \nabla\right) \boldsymbol{m}-\tilde{\beta} \frac{P_{F}}{e} \boldsymbol{m} \times\left(\boldsymbol{j}_{e} \cdot \nabla\right) \boldsymbol{m},
\end{aligned}
$$

where $\beta=\tau_{\Delta} / \tau_{s f}$ and $\tilde{\beta}=\beta /\left(1+\xi^{2}\right)$. By assuming a long dephasing time of the transverse component $\tau_{\varphi} \rightarrow \infty$ then $\xi \approx \beta$, Eq. (25) reproduces Eq. (9) in Ref. [34]. On the other hand, a short spin dephasing time $\tau_{\varphi} \rightarrow 0$ yields $\tilde{\beta} \rightarrow 0$ which results in a pure adiabatic torque, i.e., the torque reduces to the first and third terms in Eq. (25).

\section{RASHBA SPIN TORQUE}

The primary focus of this article is the Rashba torque originating from the coexistence of magnetism and Rashba spin-orbit coupling. In this section, we apply Eqs. (16) and (17) to study the properties of this torque and concentrate on possible analytical aspects in the bulk system or an infinite medium. Analytical results provide a better understanding of the physical processes behind the Rashba torque and a more transparent view on the structure of the diffusion equations derived in Sec. II. To serve this purpose, we first derive a formula that characterizes the general symmetry and angular dependence of the Rashba torque. Then for two limiting cases at weak and strong spin-orbit couplings, we are able to directly compare our results to experiments.

\section{A. General symmetry and angular dependence}

Recent studies showed that the spin-orbit torque in a ferromagnetic heterostructure possesses peculiar symmetries with respect to magnetization inversion and a complex angular dependence [26]. To be more specific, the angular dependence discussed here refers to the experimental observation that the torque amplitudes vary as functions of magnetization direction. We demonstrate in the following that such symmetries and angular dependence are encoded coherently in our simple model.

Needless to say, finding a general analytical solution to Eq. (17) with boundary conditions is by no means an easy task. But, such solutions to the spin density and spin torque do exist in an infinite medium and the behavior featured by these solutions, as the numerical solutions suggest, persists into a finite system [40]. We reorganize Eq. (17) as

$$
\frac{\boldsymbol{S}}{\tau_{s f}}+\frac{\boldsymbol{S}+S_{z} \hat{z}}{\tau_{\mathrm{dp}}}+\frac{1}{\tau_{\Delta}} \boldsymbol{S} \times \boldsymbol{m}+\frac{1}{\tau_{\varphi}} \boldsymbol{m} \times(\boldsymbol{S} \times \boldsymbol{m})=\boldsymbol{X},
$$

where the right-hand side combines the time and spatial derivatives of the spin and charge densities

$$
\begin{aligned}
\boldsymbol{X} \equiv & -\frac{\partial \boldsymbol{S}}{\partial t}+D \nabla^{2} \boldsymbol{S}+B \nabla_{z} n+2 C \boldsymbol{\nabla}_{z} \times \boldsymbol{S}+2 R\left(\boldsymbol{m} \cdot \nabla_{z} n\right) \boldsymbol{m} \\
& +\Gamma\left[\boldsymbol{m} \times\left(\nabla_{z} \times \boldsymbol{S}\right)+\nabla_{z} \times(\boldsymbol{m} \times \boldsymbol{S})\right] .
\end{aligned}
$$

A stationary state solution defined by $\partial \boldsymbol{S} / \partial t=0$ is of our current interest, whereas, in the next section, we will see that this term induces a correction contributing to the spin and charge pumping effects. In an infinite medium with an applied electric field $\boldsymbol{E}$, we again replace the spatial gradient $\boldsymbol{\nabla}$ by $\left(e / \epsilon_{F}\right) \boldsymbol{E}$ and $\boldsymbol{X}$ reduces to

$$
\begin{aligned}
\boldsymbol{X} \approx & \frac{e}{\epsilon_{F}}[B n \hat{z} \times \boldsymbol{E}+2 C(\hat{z} \times \boldsymbol{E}) \times \boldsymbol{S} \\
& +2 R n(\boldsymbol{m} \cdot(\hat{z} \times \boldsymbol{E})) \boldsymbol{m}+\Gamma \boldsymbol{m} \times((\hat{z} \times \boldsymbol{E}) \times \boldsymbol{S}) \\
& +\Gamma(\hat{z} \times \boldsymbol{E}) \times(\boldsymbol{m} \times \boldsymbol{S})],
\end{aligned}
$$

where we have discarded $\nabla^{2} S$ that is quadratic in $E$.

For a general expression Eq. (28), we may solve Eq. (26) using the partition $\boldsymbol{S}=S_{\|} \boldsymbol{m}+\delta \boldsymbol{S}$. A lengthy algebra leads to

$$
\begin{aligned}
S_{\|}= & \tau_{\mathrm{dp}} \gamma_{\theta} \chi_{\theta} \boldsymbol{X} \cdot\left[\boldsymbol{m}\left(1+\xi \chi \tilde{\beta} \sin ^{2} \theta\right)\right. \\
& \left.-\chi \tilde{\beta} m_{z}(\xi \boldsymbol{m} \times \hat{z} \times \boldsymbol{m}+\hat{z} \times \boldsymbol{m})\right],
\end{aligned}
$$

$$
\begin{aligned}
\delta \boldsymbol{S}= & \frac{\tau_{\Delta} \gamma_{\theta}}{1+\xi^{2}}[\boldsymbol{m} \times \boldsymbol{X}+\xi \boldsymbol{m} \times \boldsymbol{X} \times \boldsymbol{m} \\
& -\xi \chi_{\theta} m_{z}(\boldsymbol{X} \cdot \boldsymbol{m}) \boldsymbol{m} \times \hat{z} \times \boldsymbol{m} \\
& \left.+\chi_{\theta}\left(m_{z} \boldsymbol{X} \cdot \boldsymbol{m}+\beta \boldsymbol{X} \cdot(\hat{z} \times \boldsymbol{m})\right) \hat{z} \times \boldsymbol{m}\right],
\end{aligned}
$$

where $\theta$ is defined as the azimuthal angle between $\boldsymbol{m}$ and $\hat{z}$ and

$$
\begin{aligned}
\chi & =\frac{\tau_{s f}}{\tau_{s f}+\tau_{\mathrm{dp}}}, \\
\chi_{\theta} & =\frac{\chi}{1+\chi \cos ^{2} \theta}, \\
\gamma_{\theta} & =\frac{1}{1+\xi \tilde{\beta} \chi_{\theta} \sin ^{2} \theta} .
\end{aligned}
$$

The results of spin density in Eq. (30) give rise to the spin torque defined by Eq. (24). In an infinite medium, the spin torque reads

$$
\begin{aligned}
\boldsymbol{T}= & \gamma_{\theta}[(1-\xi \tilde{\beta}) \boldsymbol{m} \times \boldsymbol{X} \times \boldsymbol{m}+\tilde{\beta} \boldsymbol{m} \times \boldsymbol{X} \\
& +\tilde{\beta} \chi_{\theta}\left[(\xi-\beta) \boldsymbol{X} \cdot(\hat{z} \times \boldsymbol{m})-m_{z}(\boldsymbol{X} \cdot \boldsymbol{m})\right] \hat{z} \times \boldsymbol{m} \\
& \left.-\chi_{\theta}\left[(1-\xi \tilde{\beta}) m_{z} \boldsymbol{X} \cdot \boldsymbol{m}+\tilde{\beta} \boldsymbol{X} \cdot(\hat{z} \times \boldsymbol{m})\right] \boldsymbol{m} \times \hat{z} \times \boldsymbol{m}\right],
\end{aligned}
$$

which clearly exhibits two outstanding features. First, it is possible to divide the torque into two components that are either odd or even with respect to inversion of magnetization direction. Second, every component has a pronounced angular dependence.

The formulation of Eq. (32) motivates us to attempt an interpretation of $\boldsymbol{X}$ as a source term, which allows us to extend the applicability of Eq. (32) to include other driving mechanisms due to temporal variation or magnetic texture. We may further simplify Eq. (28) by observing that all 
nonequilibrium spin and charge densities shall-in the lowest order-be linear in $E$. As we are only interested in the linear response regime, we can approximate $S$ by an equilibrium value $n P_{F} \boldsymbol{m}$ and the source term becomes

$$
\begin{aligned}
\boldsymbol{X} \approx & e \mathcal{N}_{F}\left[B \hat{z} \times \boldsymbol{E}+2 C P_{F}(\hat{z} \times \boldsymbol{E}) \times \boldsymbol{m}\right. \\
& \left.+2 R(\boldsymbol{m} \cdot(\hat{z} \times \boldsymbol{E})) \boldsymbol{m}+\Gamma P_{F} \boldsymbol{m} \times((\hat{z} \times \boldsymbol{E}) \times \boldsymbol{m})\right],
\end{aligned}
$$

which serves as a starting point of the following discussions on spin torques in two major limits.

\section{B. Weak spin-orbit coupling}

In our system, a weak Rashba spin-orbit coupling implies a low D'yakonov-Perel relaxation rate $1 / \tau_{\mathrm{dp}} \propto \alpha^{2}$ such that $\tau_{\mathrm{dp}} \gg \tau_{s f}, \tau_{\Delta}$, indicating the spin relaxation is dominated by random magnetic impurities. In this regime, spin precession about the total field dominates the electrical spin generation; we may retain only $C$ and $\Gamma$ terms in Eq. (33) and discard $B$ and $R$ terms that are of higher order in $\alpha$. Therefore, when an electric field is applied along the $\hat{x}$ direction, Eq. (33) becomes

$$
\boldsymbol{X} \approx e \mathcal{N}_{F} P_{F} E[2 C \hat{\boldsymbol{y}} \times \boldsymbol{m}+\Gamma \boldsymbol{m} \times(\hat{\boldsymbol{y}} \times \boldsymbol{m})]
$$

and the torque given in Eq. (32) reduces to a commonly accepted form

$$
\boldsymbol{T}=T_{\perp} \hat{\boldsymbol{y}} \times \boldsymbol{m}+T_{\|} \boldsymbol{m} \times(\hat{\boldsymbol{y}} \times \boldsymbol{m}),
$$

consisting of both out-of-plane $\left(T_{\perp}\right)$ and in-plane $\left(T_{\|}\right)$components with magnitudes determined by

$$
\begin{aligned}
& T_{\perp}=e E P_{F} \mathcal{N}_{F}[2(1-\xi \tilde{\beta}) C+\tilde{\beta} \Gamma], \\
& T_{\|}=e E P_{F} \mathcal{N}_{F}[(1-\xi \tilde{\beta}) \Gamma-2 \tilde{\beta} C] .
\end{aligned}
$$

Note that the in-plane torque in Eq. (37) may experience a sign flip, depending on the competition between spin relaxation and precession.

To compare directly with the results in Ref. [21], we allow $\tau_{s f} \rightarrow \infty$ and $\tau_{\varphi} \rightarrow \infty$, then $\beta \approx 0$. Under these assumptions, we have $T_{\perp} \approx 2 e E P_{F} \mathcal{N}_{F} C$ and $T_{\|} \approx e E P_{F} \mathcal{N}_{F} \Gamma$. In the dirty limit, $\Gamma \ll C$ due to $\Delta_{x c} \tau \ll 1$. By making use of the relation for the polarization $P_{F}=\Delta_{x c} / \epsilon_{F}$ and the Drude relation $j_{e}=$ $e^{2} n \tau E / m$, we obtain the out-of-plane torque

$$
\boldsymbol{T}=2 \frac{\alpha m \Delta_{x c}}{e \epsilon_{F}} j_{e} \hat{\boldsymbol{y}} \times \boldsymbol{m},
$$

which agrees with the spin torque in an infinite system in the corresponding limit as derived in Ref. [21].

\section{Strong spin-orbit coupling}

In the presence of a strong spin-orbit coupling, two effects are dominating: electric generation of spin density [2] and D'yakonov-Perel spin relaxation mechanism [27]. As the electric field is aligned along the $\hat{\boldsymbol{x}}$ direction, $\boldsymbol{X}$ is simplified to be

$$
\begin{aligned}
\boldsymbol{X} \approx & e \mathcal{N}_{F} E\left[B \hat{\boldsymbol{y}}+2 C P_{F} \hat{\boldsymbol{y}} \times \boldsymbol{m}+2 R m_{y} \boldsymbol{m}\right. \\
& \left.+\Gamma P_{F} \boldsymbol{m} \times(\hat{\boldsymbol{y}} \times \boldsymbol{m})\right],
\end{aligned}
$$

and the corresponding spin torque is

$$
\begin{aligned}
\boldsymbol{T}= & \gamma_{\theta}\left(T_{\perp}^{0} \hat{\boldsymbol{y}} \times \boldsymbol{m}+T_{\|}^{0} \boldsymbol{m} \times \hat{\boldsymbol{y}} \times \boldsymbol{m}\right) \\
& +\gamma_{\theta} \chi_{\theta}\left(T_{\perp}^{x} m_{x}+T_{\perp}^{y z} m_{y} m_{z}\right) \hat{\boldsymbol{z}} \times \boldsymbol{m} \\
& +\gamma_{\theta} \chi_{\theta}\left(T_{\|}^{x} m_{x}+T_{\|}^{y z} m_{y} m_{z}\right) \boldsymbol{m} \times(\hat{z} \times \boldsymbol{m}),
\end{aligned}
$$

where the torque amplitude parameters are defined as

$$
\begin{aligned}
T_{\|}^{0} & =e E \mathcal{N}_{F}\left[2(1-\xi \tilde{\beta}) C P_{F}+\tilde{\beta}\left(B+\Gamma P_{F}\right)\right], \\
T_{\perp}^{0} & =e E \mathcal{N}_{F}\left[-2 \tilde{\beta} C P_{F}+(1-\xi \tilde{\beta})\left(B+\Gamma P_{F}\right)\right], \\
T_{\|}^{x} & =-e E \mathcal{N}_{F} \tilde{\beta}\left(B+\Gamma P_{F}\right), \\
T_{\|}^{y z} & =e E \mathcal{N}_{F}\left[2 \tilde{\beta} C P_{F}-(1-\xi \tilde{\beta})(B+2 R)\right], \\
T_{\perp}^{x} & =-e E \mathcal{N}_{F} \tilde{\beta}(\xi-\beta)\left(B+\Gamma P_{F}\right), \\
T_{\perp}^{y z} & =-e E \mathcal{N}_{F}\left[2(\xi-\beta) C P_{F}+(1-\xi \tilde{\beta})(B+2 R)\right] .
\end{aligned}
$$

Equation (40) comprises another major result of this paper. The first term, an out-of-plane torque, can be understood as a fieldlike torque produced through the ferromagnet and the spin density generated by the inverse spin-galvanic effect. The second term, an in-plane torque, originates from the Slonczewski-Berger-type spin-transfer torque that requires spin dephasing of the transverse component of spin density. The last two terms $\hat{z} \times \boldsymbol{m}$ and $\boldsymbol{m} \times(\hat{z} \times \boldsymbol{m})$ are governed by the anisotropy in spin relaxation which allows the generation of spin density components to be perpendicular to both $\boldsymbol{m}$ and the effective Rashba field. In general, the relative magnitude of these different terms are material dependent.

In fact, the symmetry reflected in Eq. (40) compares favorably to the spin torque formula proposed based on experiments by Garello et al. [26]. More interestingly, if we allow the anisotropy in spin relaxation time to vanish by taking $\tau_{\mathrm{dp}} \gg \tau_{s f}$, Eq. (40) reduces to the form of Eq. (35) consisting only of the in-plane and out-of-plane components, whereas the complex angular dependence diminishes accordingly. This is a strong indication that this angular dependence discovered in our model arises from the anisotropic spin relaxation. Meanwhile, such an angular dependence obtained here in an infinite medium persists into a realistic experimental setup with boundaries and it is insensitive to the change in sample size [40].

\section{NUMERICAL RESULTS}

In previous sections, analytical results for the spin density and Rashba torque were obtained in various limits with respect to the relative magnitude between the spin-orbit coupling and exchange field. In this section, we numerically solve Eqs. (16) and (17) to demonstrate that they provide a coherent framework to describe the spin dynamics as well as spin torques in the diffusive regime for a wider range of parameters. Here, we consider an in-plane magnetization that lies along the $\hat{\boldsymbol{x}}$ direction and another case where the magnetization is perpendicular to the thin film plane is reported elsewhere [23]. For such a two-dimensional electron system, we adopt the following boundary conditions. First, we enforce a vanishing spin accumulation at the edges along the transverse direction, i.e., $S(y=0, L)=0$. This condition implies a strong spin-flip scattering at the edges, which is consistent with the experimental observations in spin-Hall effect [6]. Second, an electric field is applied along the $\hat{\boldsymbol{x}}$ direction; therefore, we set 

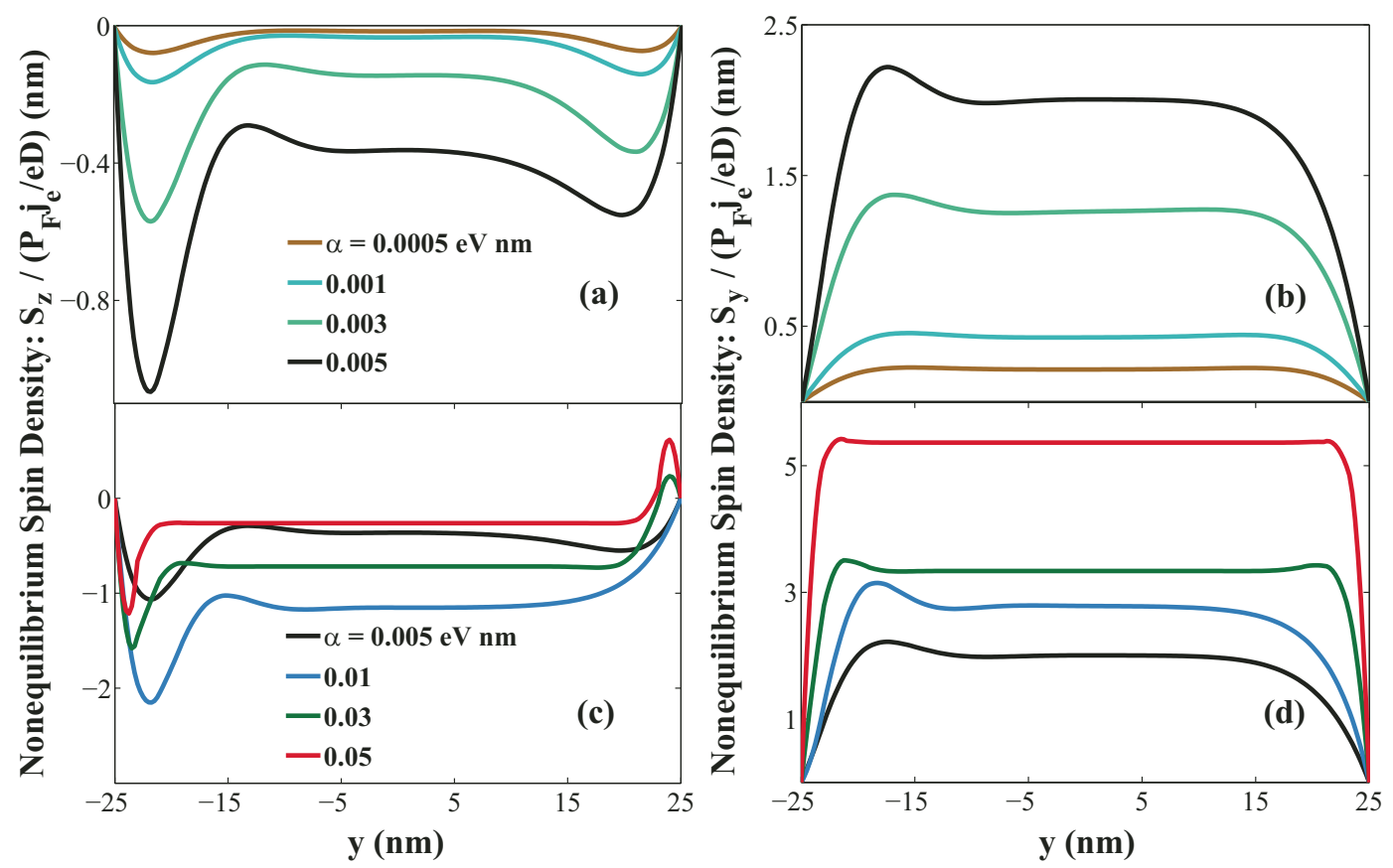

FIG. 2. (Color online) Spatial profile of the nonequilibrium spin density $S_{z}$ (a) and (c) and $S_{y}$ (b) and (d) for various values of the Rashba constant. The width of the wire is $L=50 \mathrm{~nm}$. The magnetization direction is along the $\hat{\boldsymbol{x}}$ axis. Other parameters are momentum relaxation time $\tau=10^{-15} \mathrm{~s}$, exchange splitting $\tau_{\Delta}=10^{-14} \mathrm{~s}$, spin relaxation time $\tau_{s f}=10^{-12} \mathrm{~s}$, and the Fermi vector $k_{F}=4.3 \mathrm{~nm}^{-1}$.

the charge densities at two ends of the propagation direction to be constant $n_{L}=n_{R}=n_{F}$. The second boundary condition sets the charge density at the Fermi level. Equivalently, one can apply a voltage drop along the transport direction instead of an explicit inclusion of an electric field.

The numerical results of the spin densities are summarized in Fig. 2. From the top panels [(a) and (b)] to the lower ones [(c) and (d)], for a fixed exchange splitting, the system transitions from a weak (spin-orbit-) coupling regime to a strong coupling regime. To illustrate this transition, the $S_{z}$ component of the spin density evolves from a symmetric spatial distribution in the weak spin-orbit-coupling regime, with $\alpha=5 \times 10^{-4} \mathrm{eV} \mathrm{nm}$ in Fig. 1(a), to an antisymmetric spatial distribution in the strong coupling regime, with $\alpha=5 \times 10^{-2} \mathrm{eV} \mathrm{nm}$ in Fig. 1(c). Note that throughout this transition, the in-plane spin density $S_{y}$ is robust yet roughly constant in the bulk.

This change in symmetry and the emergence of peaks close to the boundaries are resulting from the competition between the Rashba and exchange fields. In the weak coupling regime, the total field is dominated by the exchange field pointing at the $\hat{\boldsymbol{x}}$ direction, about which the spin density profile is symmetric in space. As the spin-orbit coupling increases, the total field is tilted towards the $\hat{\boldsymbol{y}}$ axis; then the spin projections along $+y$ and $-y$ are no longer symmetric, as indicted by curves with intermediate $\alpha$ values in Figs. 2(a) and 2(b). In the strong coupling regime, when the Rashba coupling overrules the exchange field, the antisymmetric profile of $S_{z}$ and the symmetric one of $S_{y}$ follow naturally from the spin-Hall effect induced by the spin-orbit interaction.

The out-of-plane and in-plane torques are plotted in Fig. 3 with respect to the Rashba constant $\alpha$ for various exchange splittings. The transition regions are of particular interest.
During the transition from a weak to strong coupling, see Fig. 3(a), the magnitude of the out-of-plane torque $T_{\perp}$ first reaches a plateau, then rises again as $\alpha$ increases. In the large $\alpha$ limit, though the magnitude of the torque increases with $\alpha$, the torque efficiency defined as $d T_{\perp} / d \alpha$ is actually smaller than it is in the weak coupling. This picture is consistent with the semiclassical Boltzmann equation description in Ref. [21]. This behavior is caused by the different processes generating the Rashba torque in both regimes. As discussed in Secs. IV B and IV C, in the weak coupling regime, the torque is dominated by the spin precession around the Rashba field, whereas in the strong coupling, the electrical generation of spin density dominates. These two distinct processes show different efficiencies.

The in-plane torque $T_{\|}$behaves differently. In the strong coupling limit, $T_{\|}$is proportional to $1 / \alpha$ due to the large D'yakonov-Perel spin relaxation rate that is of order $\alpha^{2}$. A stronger spin-orbit coupling therefore means a decrease in the torque magnitude. The transition suggests that the optimal magnitude of the in-plane torque is achieved when the exchange energy is about the same order of magnitude as the Rashba splitting $\alpha k_{F}$.

\section{DYNAMICS}

Our focus in the previous sections has always been on a stationary state with a homogeneous magnetization and the temporal and spatial variations of the ferromagnetic order parameter are neglected entirely. In this section, we demonstrate that the formulation outlined in Eq. (17) is able to address dynamic effects such as spin pumping and magnetization damping. We shall consider only the adiabatic limit where the frequency of magnetization motion is much 

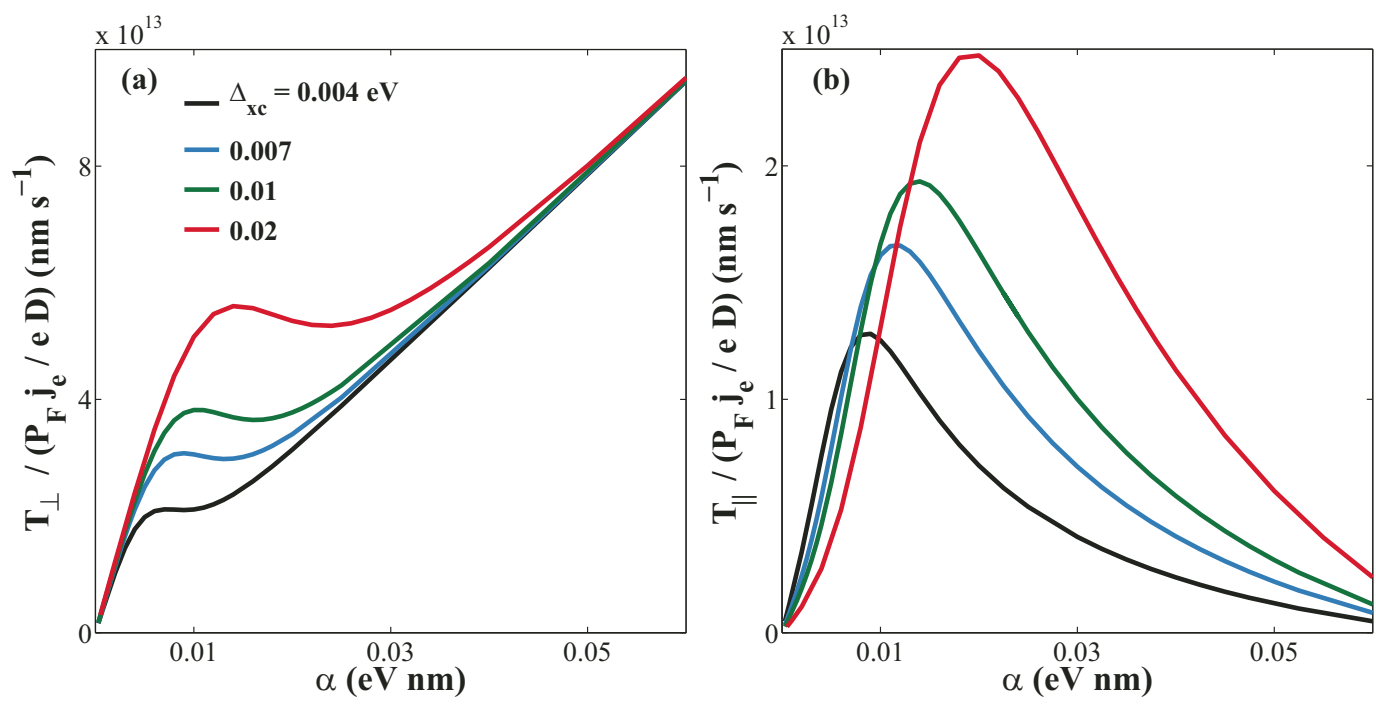

FIG. 3. (Color online) Magnitude of the out-of-plane torque $T_{\perp}$ (a) and in-plane torque $T_{\|}$(b) as a function of Rashba constant for various exchange splitting. Other parameters are the same as in Fig. 2.

lower than that of any electronic processes. Without the loss of generality, the anisotropy in spin relaxation is suppressed, for we are keen to provide a qualitative picture rather than pinpointing subtleties.

\section{A. Spin and charge pumping}

Now we consider a homogeneous single-domain ferromagnet with a moving magnetization in the absence of external electric field. In the adiabatic limit, while treating the spin-orbit coupling as a perturbation, the lowest-order correction to the spin dynamics is to let the source term

$$
\boldsymbol{X} \approx-\partial_{t} S
$$

The magnetization motion brings the system out of equilibrium and induces a nonequilibrium spin density. We can no longer naively assume that the spin density is always following the magnetization direction. To get the nonequilibrium part, we perform the usual decomposition $\boldsymbol{S}=s_{0} \boldsymbol{m}+\delta \boldsymbol{S}$ as in Sec. III B, where $\delta \boldsymbol{S}$ is referring to the nonequilibrium part induced by the magnetization motion. Here, we neglect terms like $D \nabla^{2} \delta \boldsymbol{S}$ and a simple algebra leads to

$$
\delta \boldsymbol{S}=\frac{\tau_{\Delta} s_{0}}{1+\xi^{2}}\left(\boldsymbol{m} \times \frac{d \boldsymbol{m}}{d t}+\xi \frac{d \boldsymbol{m}}{d t}\right) .
$$

Equation (43) is a formal analogy to the conventional spin pumping theory developed in magnetic multilayers using the scattering matrix approach [39,41]. Two components exist in the pumping-induced spin density and both of them are perpendicular to the magnetization direction. In the absence of spin-flip scattering $\tau_{s f} \rightarrow \infty$ thus $\xi \ll 1$ (in the dirty limit considered here), the first term $\boldsymbol{m} \times \dot{\boldsymbol{m}}$ dominates. In the conventional spin pumping theory, this contribution is governed by the real part of the spin-mixing conductance that is usually much larger than its imaginary counterpart associated with $\dot{\boldsymbol{m}}$. Equation (43) seems to suggest a similar trend. On the other hand, a strong spin-flip scattering is expected to be detrimental to the nonequilibrium spin density, which is also encoded in Eq. (43): the magnitude of $\delta \boldsymbol{S}$ decreases when the spin-flip relaxation rate $1 / \tau_{s f}$ increases.

Furthermore, the spin density induced by the magnetization motion generates a charge current via the spin galvanic effect [42], which can be estimated qualitatively to be

$$
\boldsymbol{J}_{c} \propto \frac{\alpha \tau_{\Delta} s_{0}}{1+\xi^{2}} \hat{z} \times\left(\boldsymbol{m} \times \frac{d \boldsymbol{m}}{d t}+\xi \frac{d \boldsymbol{m}}{d t}\right) .
$$

The magnitude of the charge current is proportional to the frequency of the magnetization precession.

\section{B. Magnetic damping}

When a magnetization moves in a sea of itinerant electrons, the coupling between the localized and itinerant electrons induces a friction to this motion. This friction has been described in terms of the reciprocal of the spin pumping in a magnetic texture [43]. The dynamical motion of the magnetic texture pumps a spin current that contributes to a magnetic damping when reabsorbed by the texture. In the present case, we show that the pumping of a spin-polarized current studied above can also contribute to the magnetic damping following the same process. In order to describe a magnetic texture, we allow the magnetization direction to assume a spatial dependence, i.e., $\boldsymbol{m}=\boldsymbol{m}(\boldsymbol{r})$. We limit ourselves to a weak Rashba spin-orbit coupling in order to avoid the complexity due to anisotropy in spin relaxation. For the present purpose, we keep the following source term:

$$
\boldsymbol{X}=-\partial_{t} \boldsymbol{S}+D \nabla^{2} \boldsymbol{S}+2 C(z \times \nabla) \times \boldsymbol{S} .
$$

To be more specific, we identify two sources for spatially dependent magnetic damping. One comes from the interplay between the diffusive spin dynamics and the magnetization motion, i.e., the second term in Eq. (45). The other apparently attributes to the Rashba torque, i.e., the third term in Eq. (45). We consider here an adiabatic magnetization dynamics, meaning that the electronic spin process, characterized by a time scale $\tau_{\Delta}$, is the fastest, whereas the magnetization motion, with a time scale $\tau_{M}$, is the slowest. Without loss 
of generality, we allow the spin dephasing time to sit in between, i.e., $\tau_{\Delta} \ll \tau_{\varphi} \ll \tau_{M}$. Under these assumptions, the nonequilibrium spin density pumped by the magnetization motion reads $\delta \boldsymbol{S} \approx-s_{0} \tau_{\Delta} \boldsymbol{m} \times \partial_{t} \boldsymbol{m}$ and the spatial dependent damping torques are given by

$$
\begin{gathered}
\boldsymbol{T}_{D}=-s_{0} \tau_{\Delta} D \boldsymbol{m} \times\left[\boldsymbol{m} \times \nabla^{2}\left(\boldsymbol{m} \times \partial_{t} \boldsymbol{m}\right)\right], \\
\boldsymbol{T}_{R}=2 s_{0} \tau_{\Delta} C \boldsymbol{m} \times\left\{\boldsymbol{m} \times\left[(z \times \nabla) \times\left(\boldsymbol{m} \times \partial_{t} \boldsymbol{m}\right)\right]\right\} .
\end{gathered}
$$

It is worth pointing out the symmetry properties of the last two damping torques. The damping torque due to spin diffusion, $\boldsymbol{T}_{D}$, is second order in the spatial gradient and is thus invariant under spatial inversion $\nabla \rightarrow-\nabla$. In fact, Eq. (46) has the same symmetry as the damping torque obtained by Zhang and Zhang [43]. The other damping torque that arises from the Rashba spin-orbit coupling, $\boldsymbol{T}_{R}$, is only linear in spatial gradient and is therefore referred to as chiral. In other words, in contrast to $\boldsymbol{T}_{D}$, the magnetic damping due to Rashba spin-orbit coupling is antisymmetric upon spatial inversion. Equation (47) is in agreement with the damping formula derived by Kim et al. [44]. Moreover, a more complex angular dependence of the damping coefficient emerges when the D'yakonov-Perel anisotropic spin relaxation is taken into account.

\section{DISCUSSION}

Current-induced magnetization dynamics in a single ferromagnetic layer has been observed in various structures that involve interfaces between transition metal ferromagnets, heavy metals, and/or metal-oxide insulators. Existing experimental systems are $\mathrm{Pt} / \mathrm{Co} / \mathrm{AlO}_{x}[15,16,18,19], \mathrm{Ta} / \mathrm{CoFeB} / \mathrm{MgO}$ [17], and Pt/NiFe and Pt/Co bilayers [28], as well as dilute magnetic semiconductors such as (Ga,Mn)As [12,13]. Besides the structural complexity in such systems, an unclear picture of spin-orbit coupling in the bulk as well as at interfaces places a challenge to unravel the nature of spin-orbit torque.

\section{A. Validity of Rashba model in realistic interfaces}

The well-known Rashba-type effective interfacial spinorbit Hamiltonian was pioneered by E. I. Rashba to model the influence of asymmetric interfaces in semiconducting two-dimensional electron gas [20]: a sharp potential drop, emerging at the interface (say, in the $x-y$ plane) between two materials, gives rise to a potential gradient $\nabla V$ that is normal to the interface, i.e., $\nabla V \approx \xi_{\text {so }}(\boldsymbol{r}) \hat{z}$. In case a rotational symmetry exists in-plane, a spherical Fermi surface assumption allows the spin-orbit interaction Hamiltonian to have the form $\hat{H}_{R}=\alpha \hat{\boldsymbol{\sigma}} \cdot(\boldsymbol{p} \times \hat{\boldsymbol{z}})$, where $\alpha \approx\langle\xi\rangle / 4 m^{2} c^{2}$. As a matter of fact, in semiconducting interfaces where the transport is described by a limited number of bands around a high symmetry point, the Rashba form can be recovered by $\boldsymbol{k} \cdot \boldsymbol{p}$ theory [45].

However, one can properly question the validity of the simple Rashba spin-orbit-coupling model for interfaces involving heavy metals and ferromagnets, where the band structure and Fermi surfaces are much more complex than low doped semiconducting two-dimensional electron gases for which it was initially proposed. Nonetheless, the existence of a symmetry breaking-induced spin splitting of the Rashba type has been well established by angle-resolved photoemission spectroscopy in systems consisting of a wide variety of metallic surfaces [46-48], quantum wells [49], and even oxide heterointerfaces [50]. Several important works published in the past few years on spin spirals induced by DzyaloshinskiiMoriya interaction at $\mathrm{W} / \mathrm{Fe}$ and $\mathrm{W} / \mathrm{Mn}$ interfaces [51] also argue in favor of the presence of a sizable Rashba-type spin-orbit coupling.

Besides the aforementioned experimental investigations performed on clean and epitaxially grown systems, efforts in numerical calculations have been made to the identification of an asymmetric spin splitting in the band structure of conventional metallic interfaces and surfaces. It is rather intriguing to observe that, in spite of the complexity of the band structure arising from complex hybridization among $s$, $p$, and $d$ orbitals, first principle calculations do observe such a $k$-antisymmetric spin splitting in the energy dispersion of interfacial states [52-54]. Although this spin splitting is more subtle that the simple Rashba model depicted in Eq. (1), it tends to confirm the phenomenological intuition of Rashba [20] at metallic interfaces.

\section{B. Comparison between SHE torque and Rashba torque}

At this stage, it is interesting to compare the parameter dependence of the in-plane torque $T_{\|}$[in Eq. (37)] and the torque generated by a spin-Hall effect [4] in the bulk of a heavy metal material such as Pt. In the latter case, the torque $\boldsymbol{T}^{(\mathrm{SH})}$ exerted on the normal metal/ferromagnet interface is obtained by projecting out the spin current $\left(\boldsymbol{j}^{(\mathrm{SH})}\right.$ due to spin-Hall effect) that is transverse to the magnetization direction [28]. In the bulk, the spin current can be estimated using the ratio between spin-Hall $\left(\sigma^{\mathrm{SH}}\right)$ and longitudinal $\left(\sigma_{x x}\right)$ conductivities (the socalled spin-Hall angle), i.e.,

$$
j^{(\mathrm{SH})}=\frac{\sigma^{\mathrm{SH}}}{\sigma_{x x}} j_{e} .
$$

A perturbation calculation using the second-order Born approximation gives rise to a spin current; thus the torque with a magnitude given by

$$
T^{(\mathrm{SH})}=\frac{\eta_{\mathrm{so}} m \gamma}{2 e \tau_{\mathrm{tr}}^{0}} j_{e}
$$

where, in general, $\gamma>1$ is a dimensionless parameter taking into account both side-jump $(\gamma=1)$ and skew-scattering $(\gamma>1)$ contributions to the spin-Hall effect [55]. $\eta_{\text {so }}$ is the spin-orbit-coupling parameter and $\tau_{\mathrm{tr}}^{0}$ is the transport relaxation time due to bulk impurities, the same definitions as in Ref. [55] except here the definition of spin current differs by a unit $1 /(2 e)$. Meanwhile, the magnitude of the Rashba-induced in-plane torque, i.e., Eq. (37), can be simplified to, since $\Delta_{x c} \tau \ll 1$,

$$
T_{\|} \approx \frac{4 \alpha m}{\epsilon_{F} e \tau_{s f}} j_{e} .
$$

The spin-orbit-coupling parameter $\alpha$ in our definition in Eq. (1) has the unit of energy. Equations (50) and (49) actually show that the in-plane Rashba torque and the spin-Hall torque have a very similar parameter dependence. Meanwhile, a diffusive description of the bilayer system, consisting of ferromagnet/heavy metal, has shown that both SHE torque 
and Rashba torque adopt a similar form,

$$
\boldsymbol{T}=T_{\|} \boldsymbol{m} \times(\hat{\boldsymbol{y}} \times \boldsymbol{m})+T_{\perp} \hat{\boldsymbol{y}} \times \boldsymbol{m} .
$$

The similarity in the geometrical form of the two torques implies that, in principle, they are able to induce the same type of magnetic excitation [56].

The complexity of the underlying physics of spin-orbit torque and the geometrical similarity between spin-Hallinduced and Rashba torque make it a challenge to distinguish between two possible origins. Recent progress has been made towards a plausible distinction between the bulk and interfacial origin of different torque components by varying the bilayer thickness [57], decoupling the heavy metal from the ferromagnet [58], or dusting the interface with impurities [59], which has revealed additional complex behaviors that question current models including the Rashba model.

\section{CONCLUSION}

Using Keldysh technique, in the presence of both magnetism and a Rashba spin-orbit coupling, we derive a spin diffusion equation that provides a coherent description to the diffusive spin dynamics. In particular, we have derived a general analytical expression for the Rashba torque in the bulk of a ferromagnetic metal layer in both weak and strong Rashba limits. We find that the spin-orbit torque in general consists of not only in-plane and out-of-plane components but also a complex angular dependence, which we attribute to the anisotropic spin relaxation induced by the D'yakonov-Perel mechanism.

In the presence of magnetization dynamics, we have demonstrated that our spin diffusion equation is able to describe a wealth of phenomena including spin pumping and magnetic damping. In particular, these results are in agreement with the earlier ones derived using other methods. We have discussed the common features shared by the Rashba and SHE torques. We also expect that further investigation involving structural modification of the system shall provide a deeper knowledge on the interfacial spin-orbit interaction as well as the current-induced magnetization switching in a single ferromagnet.

\section{ACKNOWLEDGMENT}

We thank G. E. W. Bauer, H. -W. Lee, K. -J. Lee, J. Sinova, M. D. Stiles, X. Waintal, and S. Zhang for numerous stimulating discussions.
[1] M. I. D'yakonov and V. I. Perel, JETP Lett. 13, 467 (1971).

[2] V. M. Edelstein, Solid State Commun. 73, 233 (1990).

[3] S. Murakami, N. Nagaosa, and S. C. Zhang, Science 301, 1348 (2003).

[4] J. E. Hirsch, Phys. Rev. Lett. 83, 1834 (1999); S. Zhang, ibid. 85, 393 (2000).

[5] J. Sinova, D. Culcer, Q. Niu, N. A. Sinitsyn, T. Jungwirth, and A. H. MacDonald, Phys. Rev. Lett. 92, 126603 (2004).

[6] Y. K. Kato, R. C. Myers, A. C. Gossard, and D. D. Awschalom, Science 306, 1910 (2004).

[7] A. S. Núñez and A. H. MacDonald, Solid State Commun. 139, 31 (2006).

[8] K. Obata and G. Tatara, Phys. Rev. B 77, 214429 (2008).

[9] J. C. Slonczewski, J. Magn. Magn. Mater. 159, L1 (1996); L. Berger, Phys. Rev. B 54, 9353 (1996).

[10] M. D. Stiles and J. Miltat, Top. Appl. Phys. 101, 225 (2006); D. C. Ralph and M. D. Stiles, J. Magn. Magn. Mater. 320, 1190 (2008); J. Z. Sun and D. C. Ralph, ibid. 320, 1227 (2008).

[11] I. Garate and A. H. MacDonald, Phys. Rev. B 80, 134403 (2009); P. M. Haney and M. D. Stiles, Phys. Rev. Lett. 105, 126602 (2010); H. Li, X. Wang, F. Dogan, and A. Manchon, Appl. Phys. Lett. 102, 192411 (2013).

[12] A. Chernyshov, M. Overby, X. Liu, J. K. Furdyna, Y. LyandaGeller, and L. P. Rokhinson, Nature Phys. 5, 656 (2009).

[13] D. Fang, H. Kurebayashi, J. Wunderlich, K. Výborný, L. P. Zârbo, R. P. Campion, A. Casiraghi, B. L. Gallagher, T. Jungwirth, and A. J. Ferguson, Nature Nanotechnol. 6, 413 (2011).

[14] M. Endo, F. Matsukura, and H. Ohno, Appl. Phys. Lett. 97, 222501 (2010).

[15] I. M. Miron, G. Gaudin, S. Auffret, B. Rodmacq, A. Schuhl, S. Pizzini, J. Vogel, and P. Gambardella, Nat. Mater. 9, 230 (2010).
[16] U. H. Pi, K. W. Kim, J. Y. Bae, S. C. Lee, Y. J. Cho, K. S. Kim, and S. Seo, Appl. Phys. Lett. 97, 162507 (2010).

[17] T. Suzuki, S. Fukami, N. Ishiwata, M. Yamanouchi, S. Ikeda, N. Kasai, and H. Ohno, Appl. Phys. Lett. 98, 142505 (2011).

[18] I. M. Miron, T. Moore, H. Szambolics, L. D. Buda-Prejbeanu, S. Auffret, B. Rodmacq, S. Pizzini, J. Vogel, M. Bonfim, A. Schuhl, and G. Gaudin, Nat. Mater. 10, 419 (2011).

[19] I. M. Miron, K. Garello, G. Gaudin, P. J. Zermatten, M. V. Costache, S. Auffret, S. Bandiera, B. Rodmacq, A. Schuhl, and P. Gambardella, Nature (London) 476, 189 (2011).

[20] Yu. A. Bychkov and E. I. Rashba, J. Phys. C: Solid State Phys. 17, 6039 (1984).

[21] A. Manchon and S. Zhang, Phys. Rev. B 78, 212405 (2008); 79, 094422 (2009).

[22] A. Matos-Abiague and R. L. Rodriguez-Suarez, Phys. Rev. B 80, 094424 (2009).

[23] X. Wang and A. Manchon, Phys. Rev. Lett. 108, 117201 (2012). In Fig. 3(a) of that paper, a typo misplaces the diffusion constant $D$ in the expression of the vertical axis.

[24] D. A. Pesin and A. H. MacDonald, Phys. Rev. B 86, 014416 (2012).

[25] E. van der Bijl and R. A. Duine, Phys. Rev. B 86, 094406 (2012).

[26] K. Garello, I. M. Miron, C. O. Avci, F. Freimuth, Y. Mokrousov, S. Blügel, S. Auffret, O. Boulle, G. Gaudin, and P. Gambardella, Nature Nanotechnol. 8, 587 (2013).

[27] M. I. D’yakonov and V. I. Perel, Sov. Phys. Solid State 13, 3023 (1972).

[28] L. Liu, T. Moriyama, D. C. Ralph, and R. A. Buhrman, Phys. Rev. Lett. 106, 036601 (2011); Science 336, 555 (2012); L. Liu, O. J. Lee, T. J. Gudmundsen, D. C. Ralph, and R. A. Buhrman, Phys. Rev. Lett. 109, 096602 (2012).

[29] P. M. Haney, H. W. Lee, K.-J. Lee, A. Manchon, and M. D. Stiles, Phys. Rev. B 87, 174411 (2013). 
[30] K. W. Kim, S. M. Seo, J. Ryu, K. J. Lee, and H. W. Lee, Phys. Rev. B 85, 180404(R) (2012); S. M. Seo, K. W. Kim, J. Ryu, H. W. Lee, and K. J. Lee, Appl. Phys. Lett. 101, 022405 (2012).

[31] J. Rammer and H. Smith, Rev. Mod. Phys. 58, 323 (1986).

[32] E. G. Mishchenko, A. V. Shytov, and B. I. Halperin, Phys. Rev. Lett. 93, 226602 (2004).

[33] J. Rammer, Quantum Field Theory of Non-equilibrium States (Cambridge University Press, Cambridge, UK, 2007).

[34] S. Zhang and Z. Li, Phys. Rev. Lett. 93, 127204 (2004).

[35] Y. Tserkovnyak, E. M. Hankiewicz, and G. Vignale, Phys. Rev. B 79, 094415 (2009).

[36] A. A. Burkov, A. S. Núñez, and A. H. MacDonald, Phys. Rev. B 70, 155308 (2004).

[37] İ. Adagideli and G. E. W. Bauer, Phys. Rev. Lett. 95, 256602 (2005).

[38] S. Zhang, P. M. Levy, and A. Fert, Phys. Rev. Lett. 88, 236601 (2002).

[39] Y. Tserkovnyak, A. Brataas, G. E. W. Bauer, and B. I. Halperin, Rev. Mod. Phys. 77, 1375 (2005).

[40] C. Ortiz-Pauyac, X. Wang, M. Chshiev, and A. Manchon, Appl. Phys. Lett. 102, 252403 (2013).

[41] Y. Tserkovnyak, A. Brataas, and G. E. W. Bauer, Phys. Rev. Lett. 88, 117601 (2002).

[42] S. D. Ganichev, E. L. Ivchenko, V. V. Belkov, S. A. Tarasenko, M. Sollinger, D. Weiss, W. Wegscheider, and W. Prettl, Nature (London) 417, 6885 (2002).

[43] S. Zhang and S. S.-L. Zhang, Phys. Rev. Lett. 102, 086601 (2009).

[44] K. W. Kim, J. H. Moon, K. J. Lee, and H. W. Lee, Phys. Rev. Lett. 108, 217202 (2012).

[45] R. Winkler, Spin-Orbit Coupling Effects in Two-Dimensional Electron and Hole Systems (Springer, Berlin, 2003).

[46] S. LaShell, B. A. McDougall, and E. Jensen, Phys. Rev. Lett. 77, 3419 (1996); F. Reinert, G. Nicolay, S. Schmidt, D. Ehm, and S. Hüfner, Phys. Rev. B 63, 115415 (2001); G. Nicolay, F. Reinert, S. Hufner, and P. Blaha, ibid. 65, 033407 (2001); M. Hoesch, M. Muntwiler, V. N. Petrov, M. Hengsberger, L. Patthey, M. Shi, M. Falub, T. Greber, and J. Osterwalder, ibid. 69, 241401(R) (2004).

[47] O. Krupin, G. Bihlmayer, K. Starke, S. Gorovikov, J. E. Prieto, K. Döbrich, S. Blügel, and G. Kaindl, Phys. Rev. B 71, 201403(R) (2005); O. Krupin, G. Bihlmayer, K. M. Döbrich, J. E. Prieto, K. Starke, S. Gorovikov, S. Blügel, S. Kevan, and G. Kaindl, New. J. Phys. 11, 013035 (2009).
[48] Ph. Hofmann, Prog. Surf. Sci. 81, 191 (2006); T. Hirahara, K. Miyamoto, I. Matsuda, T. Kadono, A. Kimura, T. Nagao, G. Bihlmayer, E. V. Chulkov, S. Qiao, K. Shimada, H. Namatame, M. Taniguchi, and S. Hasegawa, Phys. Rev. B 76, 153305 (2007); H. Mirhosseini, J. Henk, A. Ernst, S. Ostanin, C. T. Chiang, P. Yu, A. Winkelmann, and J. Kirschner, ibid. 79, 245428 (2009); A. Takayama, T. Sato, S. Souma, and T. Takahashi, Phys. Rev. Lett. 106, 166401 (2011); K. Ishizaka, M. S. Bahramy, H. Murakawa, M. Sakano, T. Shimojima, T. Sonobe, K. Koizumi, S. Shin, H. Miyahara, A. Kimura, K. Miyamoto, T. Okuda, H. Namatame, M. Taniguchi, R. Arita, N. Nagaosa, K. Kobayashi, Y. Murakami, R. Kumai, Y. Kaneko, Y. Onose, and Y. Tokura, Nat. Mater. 10, 521 (2011).

[49] A. Varykhalov, J. Sánchez-Barriga, A. M. Shikin, W. Gudat, W. Eberhardt, and O. Rader, Phys. Rev. Lett. 101, 256601 (2008); J. H. Dil, F. Meier, J. Lobo-Checa, L. Patthey, G. Bihlmayer, and J. Osterwalder, ibid. 101, 266802 (2008); A. G. Rybkin, A. M. Shikin, V. K. Adamchuk, D. Marchenko, C. Biswas, A. Varykhalov, and O. Rader, Phys. Rev. B 82, 233403 (2010).

[50] A. D. Caviglia, M. Gabay, S. Gariglio, N. Reyren, C. Cancellieri, and J. M. Triscone, Phys. Rev. Lett. 104, 126803 (2010).

[51] P. Ferriani, K. von Bergmann, E. Y. Vedmedenko, S. Heinze, M. Bode, M. Heide, G. Bihlmayer, S. Blügel, and R. Wiesendanger, Phys. Rev. Lett. 101, 027201 (2008).

[52] A. N. Chantis, K. D. Belashchenko, E. Y. Tsymbal, and M. van Schilfgaarde, Phys. Rev. Lett. 98, 046601 (2007).

[53] G. Bihlmayer, S. Blügel, and E. V. Chulkov, Phys. Rev. B 75, 195414 (2007); G. Bihlmayer, Yu. M. Koroteev, P. M. Echenique, E. V. Chulkov, and S. Blügel, Surf. Sci. 600, 3888 (2006); M. Nagano, A. Kodama, T. Shishidou, and T. Oguchi, J. Phys.: Condens. Matter 21, 064239 (2008).

[54] S. R. Park, C. H. Kim, J. Yu, J. H. Han, and C. Kim, Phys. Rev. Lett. 107, 156803 (2011); J. H. Park, C. H. Kim, H. W. Lee, and J. H. Han, Phys. Rev. B 87, 041301 (2013).

[55] S. Takahashi and S. Maekawa, J. Phys. Soc. Jpn. 77, 031009 (2008).

[56] A. Manchon, arXiv:1204.4869.

[57] J. Kim, J. Sinha, H. Hayashi, M. Yamanouchi, S. Fukami, T. Suzuki, S. Mitani, and S. Ohno, Nat. Mater. 12, 240 (2012).

[58] X. Fan, J. Wu, Y. Chen, M. J. Jerry, H. Zhang, and J. Q. Xiao, Nature Commun. 4, 1799 (2013).

[59] K. S. Ryu, L. Thomas, S. H. Yang, and S. Parkin, Nature Nanotechnol. 8, 527 (2013). 